\title{
Chromosome numbers of Euphorbia pekinensis complex in Far East Asia
}

\author{
Ki-Ryong Park*, Takahide Kurosawa ${ }^{1}$, Min-Gyeong Seo ${ }^{2}$, Bo-Eun Lee ${ }^{2}$ and Soo-Kyeong Ahn ${ }^{2}$ \\ Department of Environment and Energy Engineering, Kyungnam University, Changwon 51767, Korea \\ ${ }^{1}$ Faculty of Symbiotic Systems Science, Fukusima University, Fukushima 960-1296, Japan \\ ${ }^{2}$ Masan Muhak Girls High School, Changwon 51324, Korea
}

(Received 1 December 2017; Revised 13 December 2017; Accepted 13 December 2017)

\begin{abstract}
We report somatic chromosome numbers for three species belonging to the Euphorbia pekinensis complex distributed in Far East Asia. In E. pekinensis populations distributed in Korea, $2 n=28$ and 56 were found, while the Japanese native E. lasiocaula was also found at $2 n=28$ and 56 and the Japanese endemic E. sinanensis was found at $2 n=20$. Based on the number of chromosomes, E. lasiocaula distributed in Japan supports treatment as a variety of E. pekinensis rather than as a different species, while $E$. sinanensis should be recognized as a distinct species rather than as a variety of E. pekinensis. In the same populations of E. pekinensis and E. lasiocaula, diploid and tetraploid individuals were found, and the diversity of these chromosome numbers was consistent with the morphological diversity of these populations, suggesting the future evolutionary potential of this species.
\end{abstract}

Keywords: Euphorbia pekinensis complex, chromosome number, Euphorbia

The Euphorbia pekinensis complex, belonging to Euphorbia ser. Pekinensis (Hurusawa, 1940), consists of several species morphologically similar to E. pekinensis Rupr. (Park et al., 2002). They are E. pekinensis, E. fauriei Levl. et. Vant., E. subulatifolia Hurusawa in Korea, and E. lasiocaula Boiss. and E. sinanensis (Hurusawa) T. Kurosawa et H. Ohashi in Japan. However, these species are very similar in morphology and having a great polymorphism, they have treated as many infraspecific taxa. Recently, E. fauriei is treated as a distinct species in Korea (Park et al., 2002; Chung et al., 2003), but E. subulatifolia is morphologically very similar to $E$. pekinensis, and it treated as a Galarhoeus pekinensis var. subulatifolius (Hurusawa, 1954), or the same species as E. lasiocaula in Japan (Kurosawa and Ohashi, 1994b). In Japan, E. sinanensis, is traditionally treated as G. lasiocaulus var. sinanensis (Hurusawa, 1954) or E. pekinensis form. sinanensis (Hurusawa, 1940), and E. lasiocaula also as G. lasiocaulus form. lasiocaulus (Hurusawa, 1954), but recently both species have been recognized as an independent species based on morphological and phenological differences (Kurosawa and Ohashi, 1994a; Kurosawa, 1995).

Although few cytological studies of East Asian Euphorbia species have been conducted, two species of the E. pekinensis complex are known about chromosome numbers (Chung et al., 2003; Ikeda et al., 2008). In particular, E. fauriei was diploid with $2 n=28$, and $E$. pekinensis showed $2 n=28$ or 56 .

Therefore, this study was aimed to compare the results of somatic chromosome numbers at the population level of the E. pekinensis complex in Korea and Japan, and to utilize them as useful information for testing species limit and their relationships in this complex.

\section{Materials and Methods}

This study was carried out on 6 populations of 3 species belonging to the E. pekinensis complex distributed in Korea and Japan. The plants were collected from the natural habitats, and transplanted in the greenhouse for collecting root tips. And Voucher specimens used in this study were deposited in the herbarium of Kyungnam University (KNUH).

To investigate the number of chromosomes, chromosomes of metaphase cells were observed using the squash technique according to Chung et al. (2003) who recently observed the chromosomes of Euphorbia species. Five roots per plant

\footnotetext{
*Author for correspondence: park@kyungnam.ac.kr
} 
cultivated in pots were cut and placed in a tube containing secondary distilled water for pretreatment at $4^{\circ} \mathrm{C}$ for overnight. Roots were fixed with aceto-alcohol (1:3) for $6 \mathrm{~h}$. The fixed roots were washed with distilled water for $20 \mathrm{~min}$, and then hydrolyzed with $1 \mathrm{~N} \mathrm{HCl}$ at $60^{\circ} \mathrm{C}$ for $4 \mathrm{~min}$. After cleaning the roots with distilled water for $10 \mathrm{~min}$, stained with Feulgen reagent for $5 \mathrm{~min}$, and removed extra parts of root tips, and finally double-stained with $2 \%$ aceto-carmine and squashed. The squashed samples of five individuals per population were observed with light microscopy under $\times 1000$ magnification.

\section{Results and Discussion}

According to the results of this study, E. pekinensis individuals, which are collected from Danyang, Gotji Beach and Mt. Gahak, showed only a diploid with a chromosome number of $2 n=28$ (Table 1; Fig. 1f). However, in the case of the Daegu population, both the diploid $(2 n=28)$ and tetraploid $(2 \mathrm{n}=56)$ were occurred (Fig. $1 \mathrm{c}, \mathrm{d})$, and the Japanese native species, E. laciocaula, also showed $2 n=28$ and 56 (Fig. 1a, b) at the same population. This result is consistent with the report of the previous $E$. pekinensis chromosome study (Chung et al., 2003). They reported that E. pekinensis and E. fauriei in Korean populations were $2 n=28$, and diploid and tetraploid individuals were only found in $E$. pekinensis population. The Japanese endemic E. sinanensis was the first to report chromosomal numbers with $2 n=20$ (Fig. 1e, basic chromosome number $x=10$ ) in this study, which is a distinct difference from the rest of the E. pekinensis complex species having basic chromosome number $x=14$.

The Japanese endemic species, E. sinanensis, was treated by Hurusawa $(1940,1982)$ as a variety or forma of E. pekinensis. Recently, however, a smaller capsule and the obtuse verrucae of its surface appeared in E. sinanensis are distinct from those of E. pekinensis, and was treated as a different species by Kurosawa and Ohashi (1994a). Our results show that the two species differ in basic chromosome number as $x=14$ and 10 , supporting the treatment of Kurosawa and Ohashi (1994a) with different species rather than as infraspecific taxa of $E$. pekinensis by Hurusawa (1940, 1982). Two species have different basic chromosome numbers, and E. sinanensis can be assumed to be not closely related to the members of the $E$. pekinensis complex, and this hypothesis is consistent with the results of recent molecular studies. Phylogenetic relationships based on matK + ITS data represented that the populations of $E$. sinanensis were not placed within the E. pekinensis complex, but they showed as a sister group of the complex (Park et al., unpub. data).

Euphorbia pekinensis is widely distributed in Northeast Asia and is highly variable in morphology, so it has been confusing to taxonomic treatment, and there have been reported various subspecific taxa depending on the region. Three species of $E$. pekinensis, E. fauriei and E. subulatifolia were reported in Korea, but they have recently been treated with one species of $E$. pekinensis with three varieties. Recent systematic studies have shown that the genetic identity among three taxa is very high and may have recently been differentiated from a common ancestor (Park, 2004). In Japan, E. pekinensis and E. lasiocaula are regarded as independent species based on the difference of verrucae shapes of capsules, and also Korean endemic $E$. subulatifolia is treated as a same species of E. lasiocaula while E. fauriei is treated as a subspecies of E. pekinensis (Hurusawa, 1954; Kurosawa and Ohashi, 1994b). Traditionally, however, E. lasiocaula has been treated as the same species as $E$. pekinensis (Maximowicz, 1883; Hayata, 1904; Hara, 1935).

Although there are limitations in determining whether two species are the same species with only chromosome information, the species mentioned above showed the same number of chromosomes, and the similar variation such as occurring diploid and tetraploid individuals in a population, It would be more likely to hypothesize them as the same species rather than treating them as different species. Recent phylogenetic results based on molecular data show that these groups are combined to a single clade without resolution, which

Table 1. Species, population, sampling location and somatic chromosome number of three species in the Euphorbia pekinensis complex. The number of population examined is in parentheses.

\begin{tabular}{ccc}
\hline \hline Species & Locality & Chromosome number (2n) \\
\hline E. lasiocaula (pop 09) & Sugou, Murata-machi, Miyagi Pref., Japan & 28,56 \\
E. pekinensis (pop 01) & Mt. Gahak, Haenam, Jeonnam, Korea & 28 \\
E. pekinensis (pop 05) & Gotgi, Chungnam, Korea & 28 \\
E. pekinensis (pop 27) & Woonhung-Sa, Daegu, Korea & 28,56 \\
E. pekinensis (pop 28) & Danyang, Chungbuk, Korea & 28 \\
E. sinanensis (pop 13) & Numayama, Tsubonuma, Sendai-shi, Miyagi Pref., Japan & 20 \\
\hline
\end{tabular}



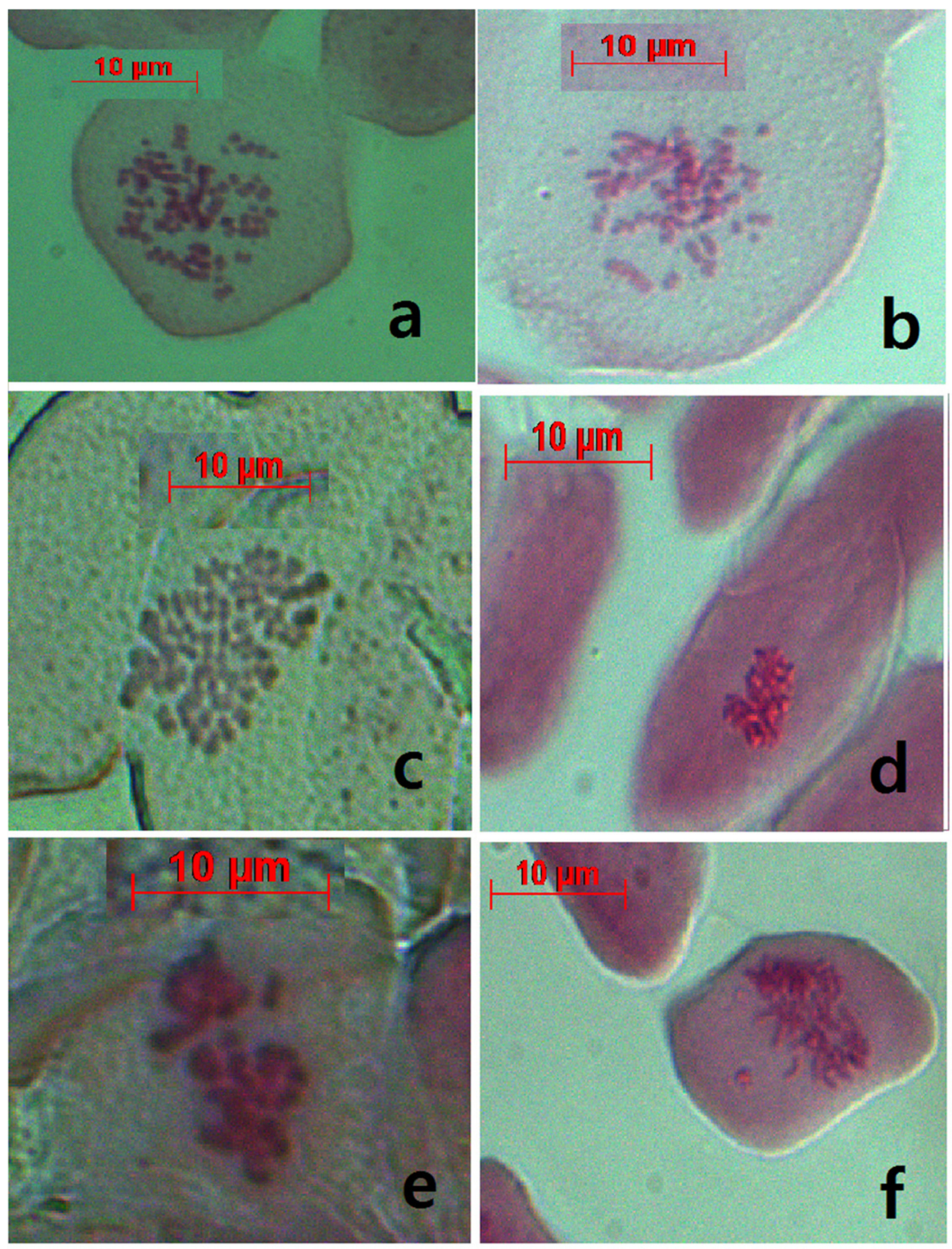

Fig. 1. Somatic chromosomes of the Euphorbia pekinensis complex species. a. E. lasiocaula (pop9, $2 n=56$ ), b. E. lasiocaula (pop9, $2 n=28$ ), c. E. pekinensis (pop27, $2 n=56$ ), d. E. pekinensis (pop27, $2 n=28$ ), e. E. sinanensis (pop13, $2 n=20$ ), f. E. pekinensis (pop1, 2n=28). Scale bars $=10 \mu \mathrm{m}$.

supports this interpretation (Park et al., unpubl. data).

The occurring of diploid and tetraploid individuals in the $E$. pekinensis complex is believed to be related to the various morphological variations within them, and they may suggest the evolutionary potential of differentiating into new species through chromosomal evolution. These similar patterns are frequently found in the Himalayan flowering plants, and are presumed to be related to the morphological variation of these plants (Wakabayashi, 2002).

\section{Acknowledgments}

We thank Dr. G. Y. Chung of Andong National University and his lab member who suggested chromosome technique, and Dr. W. J. Lee and J. H. Lee of Kyungnam University who helped us to take a photo of the light microscope. I would like to thank the reviewers for reviewing this manuscript and their suggestions. Travel expense for collecting plant samples was partially supported by the grant from National Institute of Biological Resource. 


\section{Literature Cited}

Chung, G. Y., B.-U. Oh, K.-R. Park, J.-H. Kim, M. S. Kim, G.-H. Nam and C.-G. Jang. 2003. Cytotaxonomic study of Korean Euphorbia L. (Euphorbiaceae). Korean Journal of Plant Taxonomy 33: 279-293.

Hara, H. 1935. Observationes ad plantas Asiae orientalis IV. The Journal of Japanese Botany 11: 381-390.

Hayata, B. 1904. Revisio Euphorbiacearum et Buxacearum Japonicarum. The Journal of the College of Science, Imperial University of Tokyo 20: 1-92.

Hurusawa, I. 1940. Species generis Euphorbiae imperii Japonici VI. The Journal of Japanese Botany 16: 633-645.

Hurusawa, I. 1982. Euphorbiaceae. In Wild Flowers of Japan, Herbaceous Plants (Including Dwarf Subshrubs) 2. Satake, Y., J. Ohwi, S. Kitamura, S. Watari and T. Tominari (eds.), Heibonsa Ltd., Tokyo. Pp. 224-231. (in Japanese)

Ikeda, H., T. Kurosawa and H. Ohba. 2008. Chromosome numbers and karyomorphology of three species of the genus Euphorbia L. (Euphorbiaceae) in the Sikkim Himalaya. The Journal of Japanese Botany 83: 295-300.

Kurosawa, T. 1995. Ecological differentiation of Euphorbia lasiocaula and E. sinanensis (Euphorbiaceae) I plant height, phenology and allocation to stems and leaves. Journal of Plant Research 108: 277-281.
Kurosawa, T. and H. Ohashi. 1994a. Morphological, phonological and taxonomical studies on Euphorbia lasiocaula and $E$. sinanensis (Euphorbiaceae). The Journal of Japanese Botany 69: $1-13$.

Kurosawa, T. and H. Ohashi. 1994b. Distinctions between Euphorbia lasiocaula Boiss. and E. pekinensis Rupr. (Euphorbiaceae). The Journal of Japanese Botany 69: 270-281.

Maximowicz, C. J. 1883. Diagnosesplantarum novarum Asiaticarum. V. Bulletin de l'Académie Impériale des Sciences de Saint-Pétersbourg 29: 51-227.

Park, K.-R. 2004. Comparisons of allozyme variation of narrow endemic and widespread species of Far East Euphorbia (Euphorbiaceae). Botanical Bulletin of Academia Sinica 45: 221-228.

Park, K.-R., H.-J. Jung, J.-H. Kim, G. Y. Chung and B.-U. Oh. 2002. Comparisons of morphological and genetic diversity between endemic Euphorbia fauriei and widespread E. pekinensis (Euphorbiaceae). Korean Journal of Plant Taxonomy 32: 177-191.

Wakabayashi, M. 2002. Cytological and morphological diversity in Sino-Himalayan alpine plants: Case studies in Rhodiola bupleurides and the Saxifraga pallida group. In Himalayan Botany in the Twentieth and Twenty-first Centuries. Noshiro, S. and K. R. Rajbhandari (eds.), The Society of Himalayan Botany, Tokyo. Pp. 136-140. 